12. Betrian S, Ysebaert L, Heider KH, Delord JP, Fournie JJ, QuilletMary A. Idelalisib improves CD37 antibody BI 836826 cytotoxicity against chemo-resistant/relapse-initiating CLL cells: a rationale for combination treatment. Blood. Cancer J. 2016;6: e496.
13. Krause G, Baki I, Kerwien S, Knodgen E, Neumann L, Gockeritz E, et al. Cytotoxicity of the CD37 antibody BI 836826 against chronic lymphocytic leukaemia cells in combination with chemotherapeutic agents or PI3K inhibitors. Br J Haematol. 2016; 173:791-4.

Leukemia (2019) 33:2535-2539

https://doi.org/10.1038/s41375-019-0483-z

Minimal resdiual disease

\title{
getITD for FLT3-ITD-based MRD monitoring in AML
}

\author{
Tamara J. Blätte ${ }^{1,2} \cdot$ Laura K. Schmalbrock $^{1} \cdot$ Sabrina Skambraks $^{1} \cdot$ Susanne Lux $^{1} \cdot$ Sibylle Cocciardi $^{1}$ • \\ Anna Dolnik ${ }^{1,2} \cdot$ Hartmut Döhner $^{1} \cdot$ Konstanze Döhner $^{1} \cdot$ Lars Bullinger $^{2}$
}

Received: 29 January 2019 / Revised: 20 March 2019 / Accepted: 22 March 2019 / Published online: 14 May 2019

(c) The Author(s) 2019. This article is published with open access

\section{To the Editor:}

The clinical relevance of measurable residual disease (MRD) monitoring has been well recognized in acute myeloid leukemia (AML) [1] and respective assays have been established for several recurrent leukemic markers $[2,3]$. However, although internal tandem duplications in the FLT3 gene (FLT3-ITDs) are the most common poor prognosis AML drivers [4], they have remained a challenging target: Their heterogeneity makes conventional PCRbased methods either insensitive or laborious [5]. Yet with recently approved FLT3-kinase inhibitors available [6], a specific monitoring of FLT3-mutation loads, and thus response to targeted therapy, is of particular interest. Nextgeneration sequencing (NGS) workflows for FLT3-ITD monitoring have been described, but were previously either proprietary or undisclosed $[7,8]$, unable to detect and correctly annotate all of the tested ITDs $[9,10]$, or used in

These authors contributed equally: Konstanze Döhner, Lars Bullinger

Supplementary information The online version of this article (https:// doi.org/10.1038/s41375-019-0483-z) contains supplementary material, which is available to authorized users.

Lars Bullinger

lars.bullinger@charite.de

1 Department of Internal Medicine III, University Hospital of Ulm, Ulm, Germany

2 Department of Hematology, Oncology and Tumor Immunology, Charité University Medicine, Berlin, Germany conjunction with manual analysis with inherently subjective results [10].

We have therefore developed a new method based on targeted high-coverage NGS and our novel, opensource analysis program getITD. For assay assessment, we sequenced 3 human AML cell lines (Leibniz Institute DSMZ-German Collection of Microorganisms and Cell Cultures, Braunschweig, Germany), 2 healthy volunteers, and 57 samples from 28 AML patients who were all included in the AMLSG BiO Registry study (NCT01252485) and gave their informed consent according to the Declaration of Helsinki. We show that our workflow detects ITDs of a broad range of lengths, insertion sites and variant allele frequencies (VAFs) with high accuracy and precision, is fully objective without any requirement for manual analysis, and thus applicable to routine clinical monitoring. Sample and method details are provided as supplementary information. getITD is freely available at https://github.com/tjblaette/getitd.

To demonstrate our assay's specificity, we analyzed three FLT3-ITD negative control samples (peripheral blood of healthy volunteers, $n=2$; AML cell line HL-60). No ITDs were reported, indicating an assay specificity of $100 \%$ (coverage: 1.1-4.2 million, mean 2.6 million paired-end reads). To assess sensitivity, we analyzed two serial dilutions of FLT3-ITD positive in FLT3-ITD-negative DNA from AML cell lines MOLM-14 (21 bp ITD, 67\% VAF [11]), PL-21 (126 bp ITD, 33\% VAF [11]), and HL-60 (FLT3-ITD negative [11]). For each of the ITD-positive cell lines, we sequenced undiluted DNA and 3-4 serial 1:10 dilutions in HL-60 (1.1-2.9 million, mean 2.0 million paired-end reads). The expected ITDs were detected in all samples and VAF estimates were accurate and decreased 
Fig. 1 Assessment of assay sensitivity, accuracy, and reproducibility. a DNA of FLT3 internal tandem duplication (FLT3-ITD) positive acute myeloid leukemia (AML) cell lines MOLM-14 and PL-21 was each serially diluted in DNA of the FLT3-ITD negative AML cell line HL-60; replicates were diluted independently. The expected ITDs were detected in all samples, down to a variant allele frequency (VAF) of $6.7 \times$ $10^{-5}$. Exact numbers and statistics are presented in the supplementary table S1.

b Exemplary alignments created by getITD, showing the two types of ITDs detected by our assay: a non-trailing $21 \mathrm{bp}$ ITD (left) and a trailing 198 bp ITD (right). ITD inserts are highlighted in red; the respective wild-type (WT) tandems are highlighted in green. For the trailing ITD, a lighter green mark presumably duplicated WT sequence that was not actually sequenced. Symbols "I" and "." connect matching and mismatching bases, respectively; gaps indicating insertions and deletions are annotated with "-". c Comparison of VAF estimates from PCR- and capillary electrophoresis-based fragment analysis (FA) and our nextgeneration sequencing (NGS)based assay for 28 FLT3-

ITD positive diagnostic AML samples (left) and two independent analyses of $14 / 28$ FLT3-ITD positive diagnostic AML patient samples (right). Allelic ratios (ARs) obtained by FA were converted to VAFs for this comparison as described in the supplement
A

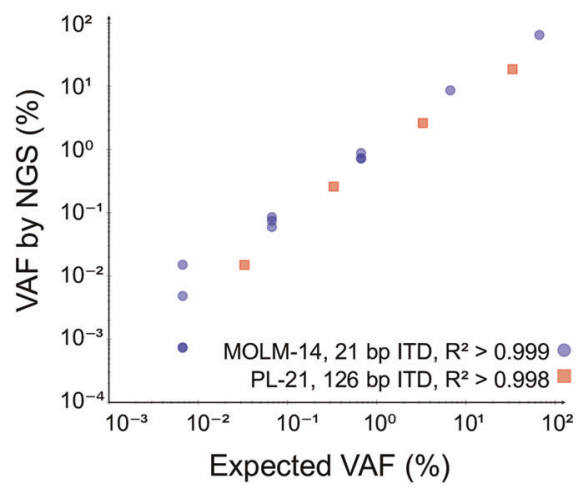

B Non-trailing ITD

Trailing ITD

ITD: GCAATTTAGGTATGAAAGCCAGCTACAGATGGTATAGGT GACCGGCTCCT

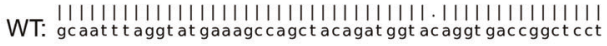

CAGATAATGAGT ACTTCTACG TTGATTTCAGAGAATATGAATTGATTTC

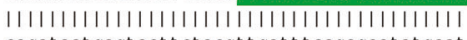
cagat aat gagt act $t$ ct acgt $t$ gat $t \mathrm{t}$ cagagaat at gaat ........

AGAGAATATGAATATGATCTCAAATGGGAGTTTCCAAGAGCAAATTTAGA ||||||||||||||||||||||||||||-||||||||| at gat ct caaat gggagt $t$ t ccaagagaaaat t t aga

GTTTGGTAAGAATGGAATGTGCCAAATGTTTCT GCAGCATTTCTTTTCCA 11111111111111111111111111111111111111111111111111 gt $t \mathrm{tggtaagaat}$ ggaat gt gccaaat gt $t \mathrm{tct}$ gcagcat $t \mathrm{tct} t \mathrm{t} t \mathrm{cca}$

TTGGAAAATCTTTAAAAT GCACGTACTCACCATTTGTCTTTGCAGGGAAG 111111111111111111111111111111111111111111111111111 $t \mathrm{tggaaaat} c t \mathrm{t} t$ aaaat gcacgt act caccat $t \mathrm{tgt}$ ct $t \mathrm{t} \mathrm{gcagggaag}$

G gt act aggat caggt gct $t \mathrm{t} t \mathrm{ggaaaagt}$ gat gaacgcaacagct $t$ at gg

ITD: GCAATTTAGGTATGAAAGCCAGCTACAGATGGTACAGGTGACCGGCTCCT

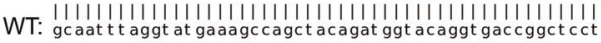

AGATAATGAGTACTTCTACGTTGATTTCAGAGAATATGAATATGATCTC

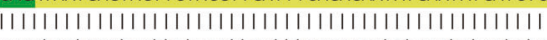
cagat aat gagt act $t$ ct acgt $t$ gat $t \mathrm{tcagagaat}$ at gaat at gat ct $c$

AAATGGGAGTTTCCAAGAGAAAATTTAGAGTTTGGTAAGAAT GGAATGTG |||||||||||||||||||||||||||||||||||||||||||||||||||| aaat gggagt $\mathrm{t} t \mathrm{cca}$ agagaaat $\mathrm{t} \mathrm{t}$ agagt $\mathrm{t} \mathrm{t}$ ggt aagaat ggaat gt $\mathrm{g}$

CCAAATGTTTCTGCAGCATTTCTTTTCCATT GGAAAATCTTTAAAATGCA |||||||||||||||||||||||||||||||||||||||||||||||||||| ccaaat gt t t ct gcagcat t t ct t t t ccat tggaaaat ct t t aaaat gca

CGTACTCACCATTTGTCTTTGCAGGGAAGCCACAGGT GACCGGCTCCTCA 1111111111111111111111111111

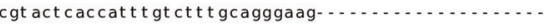

A.

C

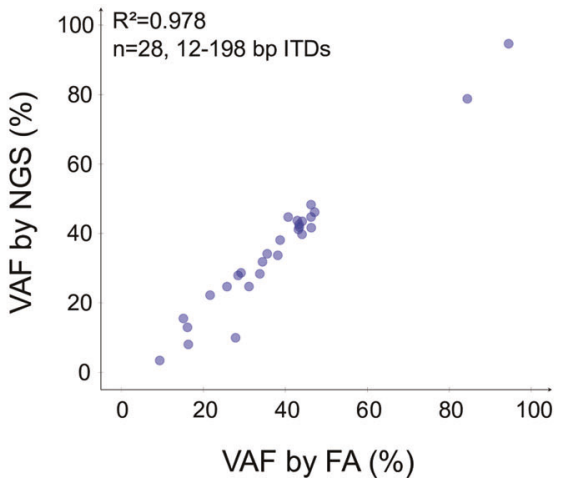

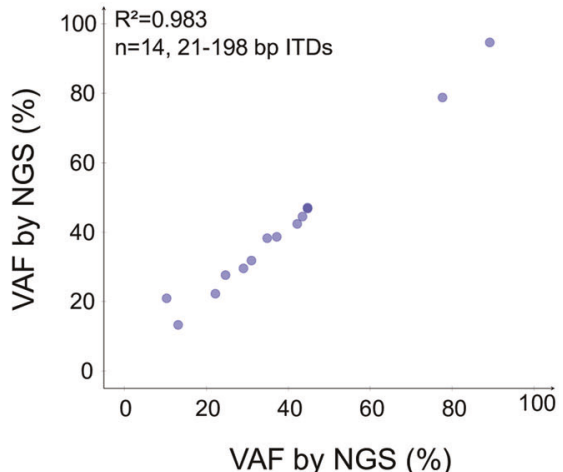

linearly as expected (MOLM-14: $R^{2}>0.999$; PL-21: $R^{2}>$ 0.998; Fig. 1a). The most diluted sample, the $1: 10,000$ MOLM-14:HL-60 dilution, harbored the ITD at $0.0067 \%$ $\operatorname{VAF}\left(6.7 \times 10^{-5}\right)$, demonstrating that our limit of detection is at least this low. Note that while MOLM-14's 21 bp ITD was correctly identified as such, PL-21's 126 bp ITD was estimated to be $125 \mathrm{bp}$ long. PL-21's ITD is too long for the insert and its wild-type (WT) tandem to be spanned by the same read $(2 \times 126 \mathrm{bp}>250 \mathrm{bp})$ and, for these trailing ITDs, length estimates are non-exact but should deviate by $\leq 3$ bp (Fig. 1b).
To determine assay accuracy, we sequenced the diagnostic FLT3-ITD positive bone marrow samples of $28 \mathrm{AML}$ patients (0.04-2.6 million, mean 1.4 million paired-end reads) and compared results to those of previously obtained fragment and Sanger sequencing analyses. In these 28 samples, PCR- and capillary electrophoresis-based fragment analysis (FA) had identified a total of 34 ITDs (1-2 per sample, mean 1.2). All 34 ITDs were also detected by our NGS-based assay. Insertion sites identified by our assay were all identical to those identified by Sanger sequencing and VAF estimates were highly correlated with 
A

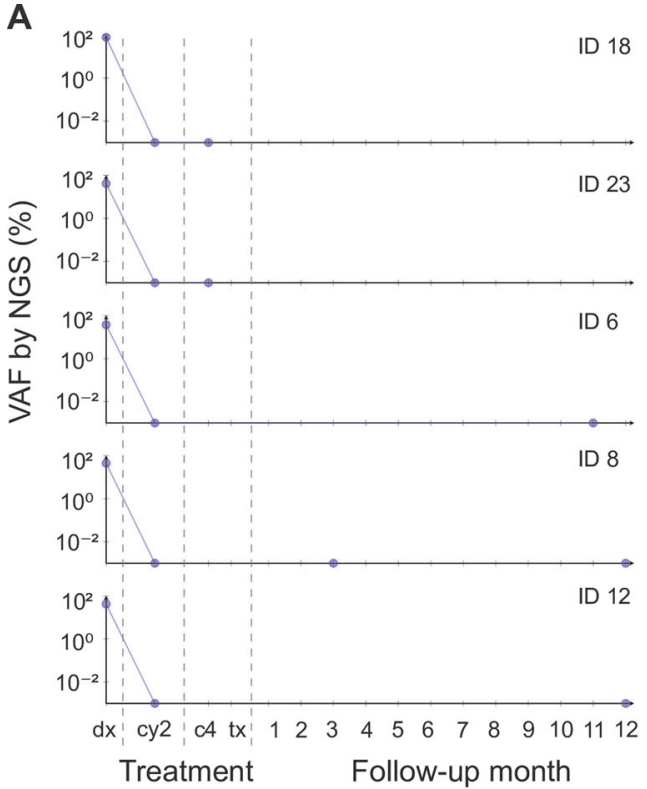

B

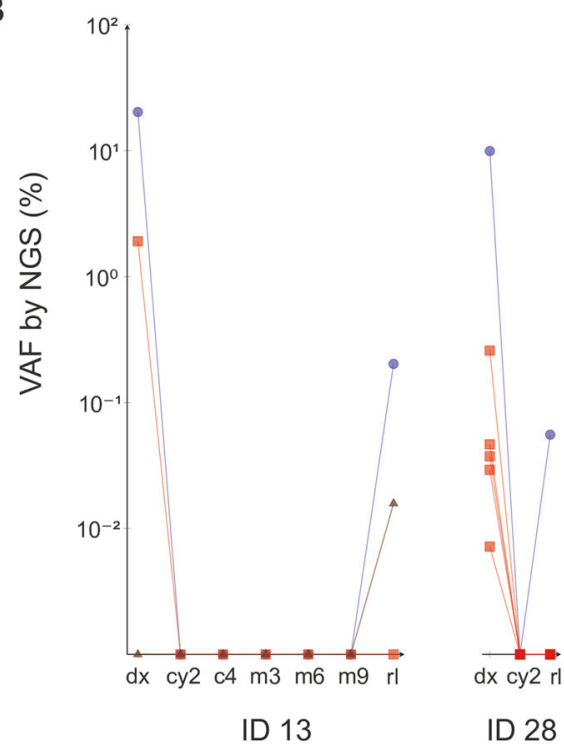

Fig. 2 Measurable residual disease (MRD) analysis in acute myeloid leukemia (AML) samples. a Serial MRD analysis of five FLT3 internal tandem duplication (FLT3-ITD) positive AML patients who remained in continuous clinical remission over the course of treatment (left) and five FLT3-ITD-positive AML patients who relapsed during follow-up (right). Each plot shows the results of a single patient: sequenced samples are marked and connected for better visibility; where applicable the relapse sample is marked in red. Marks on the $x$-axis represent samples which were analyzed but which were FLT3-ITD negative by our assay. When multiple ITD clones were detected at a certain time point, their variant allele frequencies (VAFs) were summed for plotting. b Serial MRD analysis of individual FLT3-ITD clones in the five FLT3-ITD positive AML patients who relapsed

those from FA $\left(R^{2}=0.978 ;\right.$ Fig. $\left.1 \mathrm{c}\right)$. Estimated ITD lengths were identical to those previously determined for $33 / 34$ ITDs; the $198 \mathrm{bp}$ ITD was reported as $197 \mathrm{bp}$ and trailing.
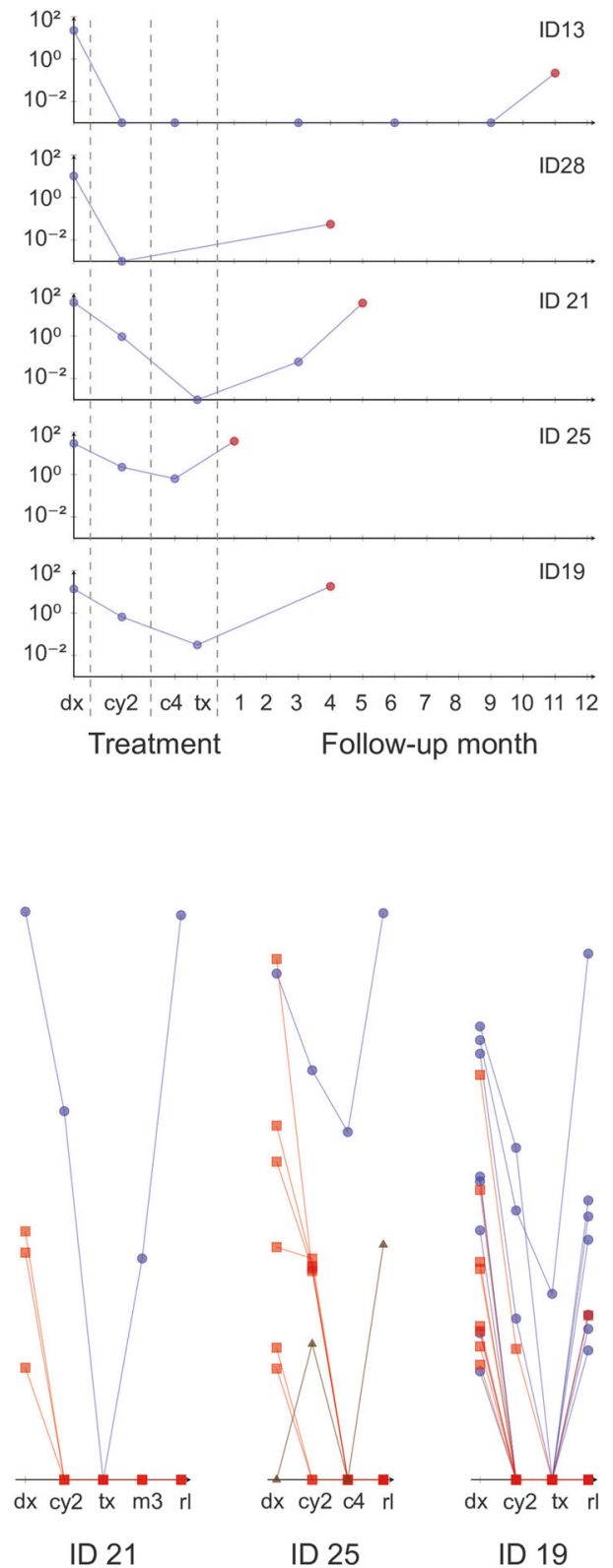

during follow-up. We analyzed the diagnosis and two to six follow-up samples, including the respective relapse sample, and plotted the change of each clone's VAF over the sampled time points. Clones persisting from diagnosis $(\mathrm{dx})$ to relapse $(\mathrm{rl})$ are shown as blue circles, clones present at diagnosis but not relapse as red squares, clones present at relapse but not diagnosis as brown triangles; no clones were detected during remission only. Marks on the $x$-axis indicate that the respective clone was not detected in that sample. Patient IDs are provided below each plot; sample, ITD, and clinical patient details are presented in the supplementary tables 2-4. Abbreviations: after 2 cycles of chemotherapy (cy2), after end of treatment: 4th consolidation treatment (c4) or stem-cell transplantation (tx), follow-up months 1-12 $(\mathrm{m} 1-12)$

In 19/28 samples, our assay identified one or more additional ITDs not detected by FA. In total, our assay detected 105 ITDs in these 28 samples (1-16 per sample, 
mean 3.8). The 71/105 ITDs, detected by our assay but not conventional FA, were similar in length to those detected by both assays (both: 12-198 bp, mean $50.4 \mathrm{bp}$; getITD only: 9-194 bp, mean $51.6 \mathrm{bp}$ ) but present at lower VAFs and thus mostly below the detection limit of conventional methods (both: 9.3-94.5\%, mean $38.2 \%$; getITD only: $0.006-2.7 \%$, mean $0.2 \%$ ). To ensure assay reproducibility, we independently re-sequenced $14 / 28$ samples at low coverage (0.2-1.3 million, mean 0.5 million paired-end reads). Results were entirely reproducible between these low- and the above-mentioned high-coverage replicates, with identical insertion sites and lengths and again highly correlated VAFs $\left(R^{2}=0.986\right.$; Fig. 1c).

To study MRD in FLT3-ITD positive AML patients, we analyzed serial samples of $10 / 28$ patients. A total of 18 follow-up samples was sequenced for five patients that relapsed during therapy (0.7-3.0 million, mean 1.8 million paired-end reads) and 11 follow-up samples were sequenced for five patients who remained in continuous complete remission (1.4-3.0 million, mean 2.1 million paired-end reads). Serial samples of patients in continuous complete remission were all FLT3-ITD negative by our assay, at all of the sampled time points (Fig. 2a, left). The relapsed patients all tested ITD positive with our assay at the time of relapse (Fig. 2a, right), whereas FA had identified only three as FLT3-ITD positive at relapse and two patients were considered to have lost the FLT3-ITD. In these two patients, our assay detected the ITDs at very low VAFs $(0.06 \%$ and $0.2 \%$, patient IDs 13 and 28, respectively; Fig. 2a), whereas no ITD could be detected in any of their preceding followup samples. For the other three relapsing patients, who tested FLT3-ITD positive at relapse also by FA, our assay detected ITDs at high VAFs at relapse (18.0-37.2\%, mean $30.3 \%$, patient IDs 21, 25, and 19; Fig. 2a) and at lower levels in at least one of the preceding follow-up samples from complete remission. Notably, in all five of the relapsed patients, there was at least one diagnostic FLT3-ITD clone that was detected also at relapse (Fig. 2b, blue circles). These ITD clones must have persisted during remission, at a VAF at times even below our limit of detection. Finally, getITD also identified, in all 10 patients, additional ITD clones that were not identified by FA: The mean number of total ITDs detected was 4.8 at diagnosis ( 1.3 by FA; $n=10$ ) and 2.7 at relapse ( 0.6 by FA, $n=5$ relapsed patients only). Those ITDs detected only by our assay were again present at very low VAFs $(0.0072-2.73 \%$, mean $0.25 \%)$ and mostly below the detection limit of conventional FA.

Importantly, sensitivity of our assay could be increased even further by applying more than the $50 \mathrm{ng}$ of sample DNA that we used. However, sensitivity will increase only linearly with input DNA, and by the same factor. In accordance, closer patient monitoring might be better suited to enable earlier MRD detection, since the amount of available patient DNA is often limited, especially during complete remission. In addition to the increased sensitivity compared to conventional FA, our approach offers the benefit of identifying ITD lengths, integration sites, and sequences, all within one assay. These are not only prognostically relevant [12-15] but also allow for the delineation and unbiased monitoring of separate ITD clones within a sample. With a current read length of $250 \mathrm{bp}$ and a minimum insert length of $6 \mathrm{bp}$, the maximum detectable ITD length of our assay would be $244 \mathrm{bp}$; longer ITDs, which are rare but do occur, would be missed. Read lengths are being extended continuously, though, and as getITD makes no assumptions on the input reads' length, it will accommodate future changes to the preceding sequencing protocol and offer a sustainable solution to the problem of FLT3-ITD detection.

NGS-based assays previously described for FLT3-ITD MRD detection used the now discontinued Roche 454 technology [7] or Illumina protocols based on random fragmentation and subsequent enrichment or enzymebased fragmentation and adapter tagging of target sequences [9, 10]. This subsequent enrichment is inefficient, leaving up to $25 \%$ of off-target reads in the mix and effectively increasing experiment costs (SureSelect Target Enrichment, Agilent Technologies Inc., Santa Clara, USA). Fragmentation of target sequences may break ITDs and render them undetectable [10]. To overcome these limitations, we used a two-step PCR to amplify and sequence the affected FLT3 exons directly. We also developed a novel analysis program, getITD, as previously described tools were either proprietary or unable to correctly identify all of the ITDs tested. Proprietary solutions generally limit transparency as well as the benefit to and from the community. It is therefore vital that code be open source, which is why getITD is freely available at https://github.com/tjblaette/getitd.

Validation by serial MRD analysis revealed persisting MRD positivity only in patients who relapsed during follow-up and additional, low-allelic FLT3-ITD clones in all patients, at diagnosis, remission and relapse, which were undetectable by FA, highlighting our method's potential for monitoring FLT3-ITD MRD in AML. In our study, our method achieved $100 \%$ sensitivity and specificity. It detected and fully annotated all of the ITDs tested and determined insertion site, length, and VAF with near perfect accuracy. Results were fully reproducible and obtained without any manual curation, making our workflow objective and easily standardized for clinical practice.

Acknowledgements We thank Annabell Markmiller for assistance with data analysis. This study was supported by grants from the 
Deutsche Krebshilfe (DKH SyTASC to LB) and the Deutsche Forschungsgemeinschaft (SFB 1074 project B3 to HD, KD and LB).

Author contributions $\mathrm{AD}, \mathrm{HD}, \mathrm{KD}$ and $\mathrm{LB}$ designed the study. TJB and SL developed program code. LKS, SS and SC developed the sequencing assay. TJB, LKS, SL, SS and SC performed experiments and data analysis. All authors contributed to and approved the final manuscript content and are fully responsible for all content and editorial decisions.

\section{Compliance with ethical standards}

Conflict of interest LB: Advisory Committees BristolMeyersSquibb, Boehringer Ingelheim, Celgene Corporation, Jazz Pharmaceuticals, Novartis, MSD Sharp \& Dohme GmbH, Merck, Seattle Genetics. HD, KD: Advisory Committees Novartis, Janssen Pharmaceutica, Daiichi Sankyo. The other authors declare that they have no conflict of interest.

Publisher's note: Springer Nature remains neutral with regard to jurisdictional claims in published maps and institutional affiliations.

Open Access This article is licensed under a Creative Commons Attribution 4.0 International License, which permits use, sharing, adaptation, distribution and reproduction in any medium or format, as long as you give appropriate credit to the original author(s) and the source, provide a link to the Creative Commons license, and indicate if changes were made. The images or other third party material in this article are included in the article's Creative Commons license, unless indicated otherwise in a credit line to the material. If material is not included in the article's Creative Commons license and your intended use is not permitted by statutory regulation or exceeds the permitted use, you will need to obtain permission directly from the copyright holder. To view a copy of this license, visit http://creativecommons. org/licenses/by/4.0/.

\section{References}

1. Schuurhuis GJ, Heuser M, Freeman S, Béné M, Buccisano F, Jacqueline Cloos, et al. Minimal/measurable residual disease in AML: consensus document from ELN MRD Working Party. Blood. 2018;131:1275-91.

2. Krauter J, Görlich K, Ottmann O, Lübbert M, Döhner H, Heit W, et al. Prognostic value of minimal residual disease quantification by real-time reverse transcriptase polymerase chain reaction in patients with core binding factor leukemias. J Clin Oncol. 2003;21:4413-22.
3. Krönke J, Schlenk RF, Jensen K, Tschürtz F, Corbacioglu A, Gaidzik VI, et al. Monitoring of minimal residual disease in NPM1-mutated acute myeloid leukemia: a study from the German-Austrian acute myeloid leukemia study group. J Clin Oncol. 2011;29:2709-16.

4. Döhner H, Weisdorf DJ, Bloomfield CD. Acute myeloid leukemia. N Engl J Med. 2015;373:1136-52.

5. Ossenkoppele G, Schuurhuis GJ. MRD in AML: does it already guide therapy decision-making? ASH Educ Program Book. 2016;1:356-65.

6. Stone RM, Mandrekar SJ, Sanford BL, Laumann K, Geyer S, Bloomfield CD, et al. Midostaurin plus chemotherapy for acute myeloid leukemia with a FLT3 mutation. $\mathrm{N}$ Engl J Med. 2017;377:454-64.

7. Thol F, Kölking B, Damm F, Reinhardt K, Klusmann J, Reinhardt $\mathrm{D}$, et al. Next-generation sequencing for minimal residual disease monitoring in acute myeloid leukemia patients with FLT3-ITD or NPM1 mutations. Genes Chromosomes Cancer. 2012;51:689-95.

8. Levis MJ, Perl AE, Altman JK, Gocke CD, Bahceci E, Hill J, et al. A next-generation sequencing-based assay for minimal residual disease assessment in AML patients with FLT3-ITD mutations. Blood Adv. 2018;2:825-31.

9. Spencer DH, Abel HJ, Lockwood CM, Payton JE, Szankasi P, Kelley TW, et al. Detection of FLT3 internal tandem duplication in targeted, short-read-length, next-generation sequencing data. J Mol Diagn. 2013;15:81-93.

10. Bibault J, Figeac M, Hélevaut N, Rodriguez C, Quief S, Sebda S, et al. Next-generation sequencing of FLT3 internal tandem duplications for minimal residual disease monitoring in acute myeloid leukemia. Oncotarget. 2015;6:22812.

11. Quentmeier H, Reinhardt J, Zaborski M, Drexler HG. FLT3 mutations in acute myeloid leukemia cell lines. Leukemia. 2003; 17:120.

12. Stirewalt DL, Kopecky KJ, Meshinchi S, Engel JH, PogosovaAgadjanyan EL, Linsley J, et al. Size of FLT3 internal tandem duplication has prognostic significance in patients with acute myeloid leukemia. Blood. 2006;107:3724-6.

13. Kayser S, Schlenk RF, Londono MC, Breitenbuecher F, Wittke K, $\mathrm{Du} \mathrm{J}$, et al. Insertion of FLT3 internal tandem duplication in the tyrosine kinase domain- 1 is associated with resistance to chemotherapy and inferior outcome. Blood. 2009;114:2386-92.

14. Schlenk RF, Kayser S, Bullinger L, Kobbe G, Casper J, Ringhoffer M, et al. Differential impact of allelic ratio and insertion site in FLT3-ITD positive AML with respect to allogeneic hematopoietic stem cell transplantation. Blood. 2014;124:3441-9.

15. Rücker FG, Du L, Blätte TJ, Benner A, Krzykalla J, Gathmann I, et al. Prognostic impact of insertion site in acute myeloid leukemia (AML) with FLT3 internal tandem duplication: results from the Ratify Study (Alliance 10603). Blood. 2018;132:435. 